\title{
Rapid and Efficient Differentiation of Rodent Neural Stem Cells into Oligodendrocyte Progenitor Cells
}

\author{
Shen $\mathrm{Li}^{\mathrm{a}, \mathrm{b}}$ Jiao Zheng ${ }^{c}$ Linlin Chai ${ }^{\mathrm{b}}$ Mengsi Lin ${ }^{\mathrm{b}}$ Ruocheng Zeng ${ }^{\mathrm{d}}$ \\ Jianhong Lu ${ }^{a, e}$ Jing Bian ${ }^{b, f}$ \\ ${ }^{a}$ Key Laboratory of Carcinogenesis of the Chinese Ministry of Health, Xiangya Hospital, Central South University, \\ Changsha, PR China; ${ }^{b}$ Key Laboratory of Neuroregeneration of Jiangsu and Ministry of Education, Co-Innovation \\ Center of Neuroregeneration, Nantong University, Nantong, PR China; ${ }^{C}$ Xijing Hospital, The Fourth Military Medical

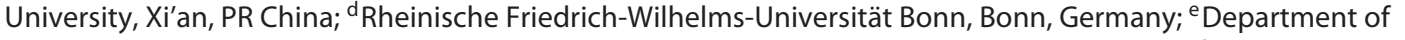 \\ Microbiology, School of Basic Medical Sciences, Central South University, Changsha, PR China; ${ }^{\mathrm{f} I n s t i t u t e}$ for Stem \\ Cell Biology and Regenerative Medicine, Stanford University, Stanford, CA, USA
}

\section{Keywords}

Oligodendrocyte progenitor cells · Oligodendrocytes ·

Neural stem cells · Neuroepithelial stem cells $\cdot$ Neural glial differentiation

\begin{abstract}
Oligodendrocyte progenitor cells (OPCs) may have beneficial effects in cell replacement therapy of neurodegenerative disease owing to their unique capability to differentiate into myelinogenic oligodendrocytes (OLs) in response to extrinsic signals. Therefore, it is of significance to establish an effective differentiation methodology to generate highly pure OPCs and OLs from some easily accessible stem cell sources. To achieve this goal, in this study, we present a rapid and efficient protocol for oligodendroglial lineage differentiation from mouse neural stem cells (NSCs), rat NSCs, or mouse embryonic stem cell-derived neuroepithelial stem cells. In a defined culture medium containing Smoothened Agonist, basic fibroblast growth factor, and platelet-derived growth factor-AA, OPCs could be generated from the above stem cells over a time course of 4-6 days, achieving a cell
\end{abstract}

\section{KARGER}

(c) 2019 S. Karger AG, Basel

E-Mail karger@karger.com

www.karger.com/dne purity as high as $\sim 90 \%$. In particular, these derived OPCs showed high expandability and could further differentiate into myelin basic protein-positive OLs within 3 days or alternatively into glial fibrillary acidic protein-positive astrocytes within 7 days. Furthermore, transplantation of rodent NSCderived OPCs into injured spinal cord indicated that it is a feasible strategy to treat spinal cord injury. Our results suggest a differentiation strategy for robust production of OPCs and OLs from rodent stem cells, which could provide an abundant OPC source for spinal cord injury.

(c) 2019 S. Karger AG, Basel

\section{Introduction}

Oligodendrocyte progenitor cells (OPCs) contribute to myelin maintenance and repair in the central nervous system (CNS) due to their unique capability to differenti-

Jianhong Lu

NHC Key Laboratory of Carcinogenesis of the Chinese Ministry of Health Xiangya Hospital, Central South University

Changsha 410080 (PR China)

E-Mail jianhlu@csu.edu.cn

Jing Bian

Institute for Stem Cell Biology and Regenerative Medicine

Stanford University, 265 Campus Drive West

Stanford, CA 94305 (USA)

E-Mail dudubian@stanford.edu 
ate into myelinogenic oligodendrocytes (OLs) in response to extrinsic signals [1]. The loss of OLs after CNS degeneration or damage (e.g., multiple sclerosis or white matter injury) may lead to many neural disorders [2-4], and current therapeutic strategies remain deficient in promoting remyelination. Cell replacement therapy with OPCs may become a promising choice because of their beneficial effects on myelin restoration [5]. Therefore, it is of significance to establish an effective methodology to differentiate reliable stem cells, such as adult neural stem cells (NSCs) or pluripotent stem cell-derived NSCs, into OPCs and mature OLs with a high purity. Although many studies have achieved some progress in this respect [6-8], those differentiation strategies still need to be improved, especially due to the formation of a heterogeneous population of uncharacterized, tripotent neural precursors rather than OPCs.

A number of studies have focused on neural differentiation of human pluripotent stem cells, but the longterm process greatly limits the application of these studies $[9,10]$. Since embryo development takes much less time in rodents than in humans, it may be relatively easy to find key inducing factors for controlling neural differentiation of rodent stem cells. Moreover, rodents are more relevant to humans, and the key factors used to regulate rodent neural differentiation would also be applicable to human neural differentiation. Unfortunately, few studies have reported on rapid generation of pure and committed OPCs derived from rodent NSCs and further differentiation of the obtained OPCs into mature OLs or astrocytes.

It has been known that most OPCs are initially differentiated from neural precursors, known as neuroepithelial stem cells (NESCs), in ventral spinal cord at embryonic day 12.5 (E12.5) to E14.5 [11]. Following the developmental principles $[3,12]$, we developed a method to generate OPCs and then produce mature OLs from mouse NSCs (mNSCs) or rat NSCs (rNSCs). We found that mNSCs, rNSCs, or mouse embryonic stem cell (mESC)derived NESCs could be induced to differentiate into OPCs using the cocktail of Sonic Hedgehog (SHH) or Smoothened Agonist (SAG), basic fibroblast growth factor (FGF2), and platelet-derived growth factor-AA (PDGF-AA), or into OLs using the cocktail of triiodothyronine (T3), SAG, and other components [13] within 7 days. RT-PCR and immunofluorescence analysis were performed to confirm the cell identity and cell purity of OPCs or OLs with representative cell markers. We postulated that precise control of these signals would allow for the routine and specific generation of sufficient quantities of OPCs from NSCs. Our in vivo experiment demonstrated that transplantation of rodent NSC-derived OPCs is a feasible strategy to treat spinal cord injury.

\section{Materials and Methods}

Cell Culture and Differentiation Medium

OPC medium was composed of Dulbecco's modified Eagle's medium/Ham's F-12 medium (DMEM/F12, Gibco) supplemented with $1 \times$ B27 (Gibco), 1× N2 (Gibco), 20 ng/mL FGF2 (Pepro Tech), $20 \mathrm{ng} / \mathrm{mL}$ PDGF-AA (Pepro Tech), and 0.4 $\mu \mathrm{M}$ SAG (Enzo Life Sciences) or $200 \mathrm{ng} / \mathrm{mL}$ SHH (R\&D Systems). OL medium was composed of DMEM/F12 supplemented with $1 \times \mathrm{B} 27,1 \times \mathrm{N} 2,30$ ng/mL T3 (Sigma), $0.4 \mu \mathrm{M}$ SAG, $100 \mathrm{ng} / \mathrm{mL}$ Noggin (R\&D Systems), $10 \mu \mathrm{M}$ dibutyryl cyclic-AMP sodium salt (Sigma), $100 \mathrm{ng} /$ $\mathrm{mL}$ IGF-1 (PROSPEC), and $10 \mathrm{ng} / \mathrm{mL}$ NT3 (R\&D Systems). The small molecules are listed in online supplementary Table S1 (see www.karger.com/doi/10.1159/000499364 for all online suppl. material).

Astro type 2 medium was composed of DMEM/F12 supplemented with $1 \times \mathrm{B} 27,1 \times \mathrm{N} 2$, and $20 \mathrm{ng} / \mathrm{mL}$ BMP4 (R\&D Systems). Astro type 1 medium was composed of DMEM/F12 supplemented with $20 \%$ fetal bovine serum (FBS; Gibco).

NE 1 medium was composed of DMEM/F12 supplemented with $1 \times$ B27, $1 \times \mathrm{N} 2,1 \times$ NEAA (Gibco), $0.1 \mathrm{mM} \beta-\mathrm{Me}$ (Gibco), 100 $\mathrm{ng} / \mathrm{mL}$ Noggin, $20 \mu \mathrm{M}$ SB431542 (Sigma), $2 \mu \mathrm{M}$ Dorsomorphin (Sigma), and $3 \mu \mathrm{M}$ CHIR99021 (Sigma). NE 2 medium was composed of DMEM/F12 supplemented with $1 \times \mathrm{N} 2,20 \mathrm{ng} / \mathrm{mL}$ FGF2, and $1.6 \mathrm{~g} / \mathrm{L}$ glucose (Sigma). NE 3 medium was composed of DMEM/F12 supplemented with $1 \times \mathrm{N} 2,1 \mu \mathrm{L} / \mathrm{mL}$ B27 (1:1,000), 20 $\mu \mathrm{g} / \mathrm{mL}$ insulin (Wako), $1.6 \mathrm{~g} / \mathrm{L}$ glucose, $20 \mathrm{ng} / \mathrm{mL}$ epidermal growth factor (EGF) (R\&D Systems), and $20 \mathrm{ng} / \mathrm{mL}$ FGF2. All cell cultures were performed at $37^{\circ} \mathrm{C}$ and $5 \% \mathrm{CO}_{2}$.

\section{Isolation and Culture of Rodent NSCs}

mNSCs were isolated from E13.5 pregnant ICR mice (Experimental Animal Center, Nantong University) as described previously [14]. In brief, animals were deeply anesthetized with an intraperitoneal injection of xylazine $(10 \mathrm{mg} / \mathrm{kg})$, ketamine $(95 \mathrm{mg} /$ $\mathrm{kg})$, and acepromazine $(0.7 \mathrm{mg} / \mathrm{kg})$. Under deep anesthetization, the animals were sacrificed, and the head of embryo was removed quickly and immerged into phosphate-buffered saline (PBS, pH 7.2). Dissected cortical or striatal germinal zone tissues were transferred to a serum-free medium and mechanically dissociated into a cell suspension with a fire-polished Pasteur pipette. After washing with PBS and centrifugation, the cells were seeded on a Petri dish in the NSC medium consisting of DMEM/F12 supplemented with $1 \times \mathrm{B} 27,20 \mathrm{ng} / \mathrm{mL}$ EGF, and $20 \mathrm{ng} / \mathrm{mL}$ FGF2, and half of the medium was changed every 2 days. rNSCs were harvested from E13.5 pregnant Sprague-Dawley rats using the same procedures as described above for mNSCs.

\section{Differentiation of Rodent NSCs into OPCs}

mNSCs or rNSCs were dissociated to a single cell suspension using Accutase (Gibco). The cells were counted and plated at $4 \times$ $10^{4}$ cells $/ \mathrm{cm}^{2}$ on plates, which were coated with $0.1 \mathrm{mg} / \mathrm{mL}$ polyL-ornithine (Sigma) and then with $10 \mu \mathrm{g} / \mathrm{mL}$ laminin (Sigma), or coated only with 5\% Matrigel (BD Biosciences). Cells were grown
80

Dev Neurosci 2019;41:79-93

DOI: $10.1159 / 000499364$
Li/Zheng/Chai/Lin/Zeng/Lu/Bian 
and the cell medium was changed every other day. After 4 days, the culture consisted of a highly pure population of proliferating OPCs, which were quickly expanded and maintained in the OPC medium and had the ability to differentiate into OLs or astrocytes. The OPCs could also be frozen or thawed with a freezing medium (DMEM/F12 supplemented with 30\% FBS and 10\% DMSO) (Sig$\mathrm{ma})$.

\section{Differentiation of OPCs into OLs or Astrocytes}

For differentiation of rodent OPCs into OLs, the cells were plated on a dish coated with PLO/laminin or Matrigel at $2 \times 10^{4}$ cells/ $\mathrm{cm}^{2}$ for 3-day culture in the OL medium. Alternatively, rodent OPCs were cultured in the Astro type 1 medium for 7 days for differentiation into astrocytes. Attempts to modulate the outcome of this differentiation paradigm included treatment with the Astro type 2 medium.

\section{Differentiation of mESCs into NESCs}

mESCs (line M1, passages 21-40) were maintained and dissociated as single cells using Accutase and cultured on 5\% Matrigelcoated plates with a feeder-free culture medium before differentiation into NESCs. This protocol consisted of three sequential differentiations under defined conditions (NE 1, NE 2, and NE 3 medium). In brief, medium was replaced by NE 1 medium on day 1. On day 4, neural rosette-like colonies were released using 1.5 $\mathrm{mg} / \mathrm{mL}$ collagenase IV (Gibco) and fed with NE 2 medium from uncoated plates in the following 4 days. On day 8 , neurospheres were collected, treated with Accutase, and transferred to Matrigelcoated plates for culture in NE 3 medium. On day 9 (1 day after replating), almost all dissociated cultures from neurospheres consisted of a highly pure population of proliferating NESCs.

For differentiation of NESCs into OPCs, these cells were plated at $4 \times 10^{4}$ cells $/ \mathrm{cm}^{2}$ on plates coated with PLO/laminin or Matrigel and cultured in the OPC medium for 6 days. mESC-derived OPCs could be further differentiated into terminal OLs or astrocytes with the same procedures as the one described above for NSCs.

\section{Growth Curve}

The cell number of OPCs at P1 was calculated based on the formula $\mathrm{Cn}=\mathrm{C}^{\mathrm{n}-1} \times \mathrm{T}^{\mathrm{n}} / \mathrm{S}^{\mathrm{n}}$, where $\mathrm{C}$ is cumulative cell number, $\mathrm{n}$ passage number, $T$ total cell number at current passage, and $S$ seeded cell number at current passage. To evaluate the cell growth rate, cells were seeded onto 24 -well plates at a density of $8 \times 10^{4}$ cells/ well. After culture for $4-5$ days, the cells at $80-90 \%$ confluence were harvested and counted with a hemocytometer. The result was recorded as the mean cell number at each time point. Data were taken from three replicates for each passage.

\section{RNA Isolation and RT-PCR}

Total RNA was prepared using an RNeasy Plus Mini Kit (QIAGEN) following the manufacturer's instructions. cDNA was generated by reverse transcription of total RNA $(1 \mu \mathrm{g})$ using a Transcriptor First Strand cDNA Synthesis Kit (Roche). PCR was carried out using DreamTaq DNA Polymerase (Thermo Scientific). The conditions and protocols of PCR were based on the product information of DreamTaq DNA Polymerase. The PCR primers are listed in online supplementary Table S3. GAPDH was used for internal normalization, and samples without reverse transcription were used as negative controls. The amplification products were analyzed by agarose gel electrophoresis.

Rapid and Efficient Differentiation of Rodent NSCs into OPCs

\section{Immunocytochemical Staining}

Cells were fixed with $4 \%$ paraformaldehyde for $15 \mathrm{~min}$ at room temperature and rinsed three times with PBS. Spheres were fixed for $45 \mathrm{~min}$ and rinsed twice with PBS. Cells were blocked for $1 \mathrm{~h}$ at room temperature with blocking buffer ( $5 \%$ goat serum $/ 0.2 \%$ Triton X-100/PBS) and incubated overnight at $4{ }^{\circ} \mathrm{C}$ with primary antibodies (online suppl. Table S2) in $2 \%$ goat serum/PBS. Importantly, Triton was omitted throughout for myelin basic protein (MBP) staining. After three rinses in PBS, Alexa Fluor secondary antibodies (Jackson ImmunoResearch 488 or 594) were applied at 1:1,000 in PBS and Hoechst 33342 staining was applied at 1:4,000 (Sigma) for $1 \mathrm{~h}$ at room temperature. The cells were viewed under microscopy (Carl Zeiss GmbH Axio Vert.A1) with appropriate filters for cell identification and counting. Confocal imaging using a scanning laser confocal microscope (TCS-NT-UV; Leica) was also performed. For percentage of induction, pictures were counted in four random fields from 2-4 independent experiments. Data were presented as means and SD.

\section{Spinal Cord Injury Model and Cell Transplantation}

Twenty male Sprague-Dawley rats with an average body weight of 180-220 g (Experimental Animal Center, Nantong University) were used. Animals were deeply anesthetized and received laminectomies in the 11 th thoracic vertebra. Contusion injury was induced using an spinal cord injury device (IH Spinal cord impactor, Precision Systems and Instrumentation) with a force of $150 \mathrm{kDynes}$ and dwell time of $2 \mathrm{~s}$. After contusion, the muscle and skin were closed in layers. All rats received manual bladder expression twice daily and were inspected for urinary tract infection, weight loss, and distress, with appropriate care as needed.

The transgenic Sprague-Dawley rats (SD-Tg[Act-EGFP]) expressing enhanced green fluorescent protein (GFP) under the chicken $\beta$-actin promoter and cytomegalovirus enhancer were kindly provided by Dr. Mao-Rong Jiang (Laboratory Animal Center, Nantong University). The GFP-positive OPCs derived from rNSCs were used for transplantation. Seven days after injury, 10 animals were anesthetized and transplanted with GFPlabeled rat OPCs using a glass micropipette (Hamilton). A total of $5 \mu \mathrm{L}$ cell suspensions $(30,000$ cells $/ \mu \mathrm{L} ; 150,000$ cells in total) per animal were acutely transplanted into the injury epicenter in the spinal cord, injected at a rate of $1 \mu \mathrm{L} / \mathrm{min}$. Ten control injured rats were injected with $5 \mu \mathrm{L}$ PBS. The postoperative care of the cell therapy and control groups was carried out as previously described.

Five weeks after cell transplantation, the animals were deeply anesthetized and perfused transcardially with $4 \%$ paraformaldehyde in PBS. Dissected spinal cords were further postfixed in $4 \%$ paraformaldehyde in $\mathrm{PB}$ at $4{ }^{\circ} \mathrm{C}$ overnight and cryoprotected in $30 \%$ sucrose for at least $48 \mathrm{~h}$. Two-centimeter blocks of the thoracic region of the cords including injury epicenters were embedded in optimal cutting temperature OCT and cryosectioned. After embedding in Tissue-Tek OCT compound, spinal cord sections were cut to a thickness of $16 \mu \mathrm{m}$. Spinal cord sections from Sprague-Dawley rats were blocked with $10 \%$ normal goat serum in PBS at room temperature for $1 \mathrm{~h}$ and then incubated with GFP and Olig2 antibodies in $1 \%$ bovine serum albumin overnight at $4{ }^{\circ} \mathrm{C}$, followed by Alexa Fluor 488-conjugated AffiniPure Goat Anti-mouse $\operatorname{lgG}$ in PBS for $1 \mathrm{~h}$ at room temperature. Some sections were counterstained with Hoechst to show nuclei. The 


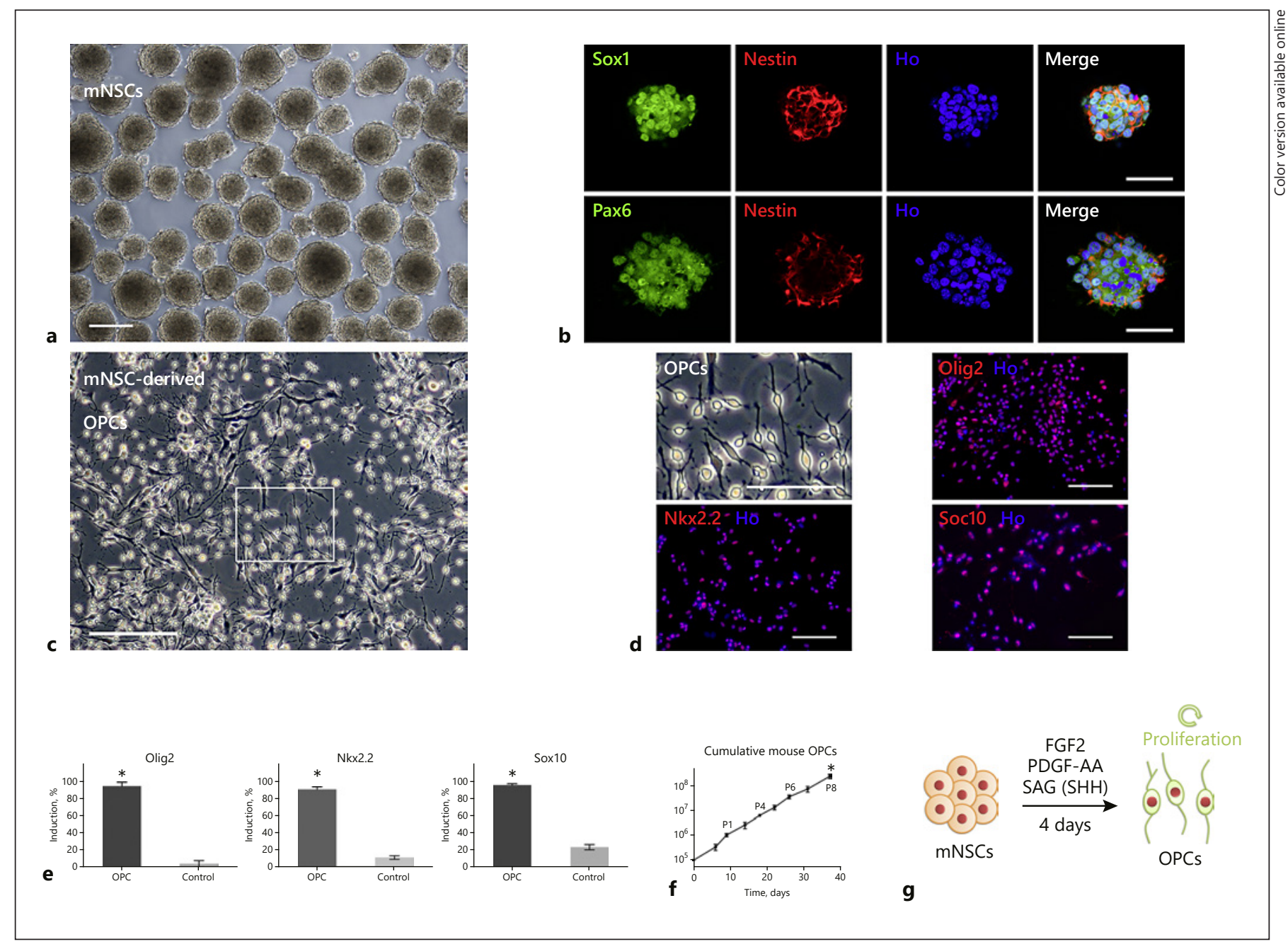

Fig. 1. Differentiation of mNSCs into OPCs treated with $\mathrm{SHH}$ FGF2 and PDGF-AA. a, b Bright-field (a) and fluorescence (b) images of NSCs isolated from mouse embryo cortex and cultured for 5 days. c Phase contrast image of formation of OPC differentiated from mNSCs on a coated dish in the presence of SHH, FGF2, and PDGF-AA. d Immunostaining of OPCs revealed that they highly expressed Olig2, Nkx2.2, and Sox10. e The percentage of Olig2-, Nkx2.2-, and Sox10-positive cells in the total population of mNSC-derived OPCs compared to negative control (mNSCs treated with medium containing only B27 and N2). f Cumulative

stained sections were examined with a confocal laser scanning microscope (Leica Microsystems, Germany).

\section{Statistics Analysis}

The quantitative data are presented as means \pm SD. Statistically significant differences between two groups were assessed by Student's $t$ test, whereas those between multiple groups were evaluated by one-way ANOVA test plus Scheffé post hoc tests with the help of SigmaStat 3.5 software (Sigma). A $p$ value $<0.05$ was considered significant. growth curves of mNSC-derived OPCs over eight passages (P1P8), P8 versus P1-P7, respectively. g Schematic representation showing the progress of differentiation of mNSCs into OPCs within 4 days. Data are expressed as means \pm SD from three independent experiments. ${ }^{*} p<0.05$. Scale bars, $200 \mu \mathrm{m}$ (a, c), $50 \mu \mathrm{m}$ (b), and $100 \mu \mathrm{m}$ (d). FGF2, basic fibroblast growth factor; Ho, Hoechst 33342-stained nuclei; mNSCs, mouse neural stem cells; NSCs, neural stem cells; OPCs, oligodendrocyte progenitor cells; PDGFAA, platelet-derived growth factor-AA; SAG, smoothened agonist; $\mathrm{SHH}$, Sonic Hedgehog.

\section{Results}

\section{Differentiation of $m$ NSCs into OPCs within 4 Days}

In the CNS, multipotent NSCs undergo self-renewal and have the potential to differentiate into neurons, astrocytes, and OLs [15]. In this study, at first, primary $\mathrm{mN}$ SCs isolated from E13.5 embryonic brain were expanded into neurospheres (Fig. 1a) under the influence of two mitogens, EGF and FGF2 [14, 16]. The neurospheres var- 


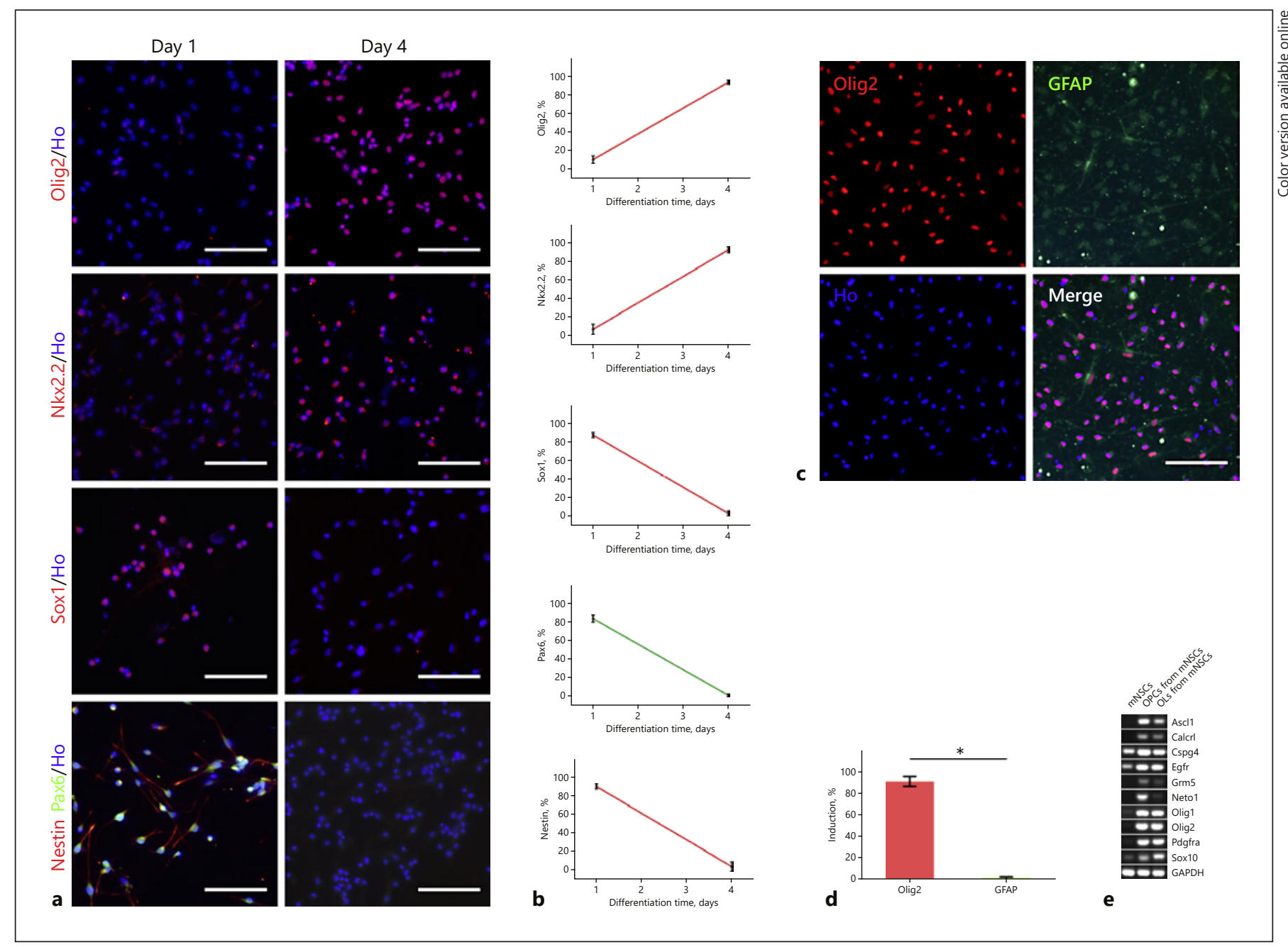

Fig. 2. Differentiation of mNSCs into OPCs within 4 days with SAG. a Immunostaining of mNSCs with neuroectodermal markers and OL lineage markers during the differentiation of mNSCs into OPCs in response to SAG, FGF2, and PDGF-AA. The treatment at day 4 resulted in the upregulation of OPC-relevant transcription factors Olig2 and Nkx2.2 and downregulated the expression of neuronal precursor markers such as Sox1, Pax6, and Nestin, as compared to treatment at day 1 . b Percentage changes of neuronal precursor markers such as Sox1, Pax6, Nestin, and OPCrelevant transcription factors Olig2 and Nkx2.2 in mNSCs under differentiation. c Immunostaining of OPCs derived from mNSCs with markers of Olig2 and GFAP. d The percentage of Olig2- and
GFAP-positive cells in the total population of mNSC-derived OPCs was $91 \pm 3 \%$ and $1 \pm 1 \%$, respectively; the value was significantly higher in the Olig2-positive population than in the GFAPpositive population. ${ }^{*} p<0.05$. e RT-PCR analysis indicated that mNSC-derived OPCs strongly upregulated OL lineage-specific genes such as Olig1/2 and Sox10 and markers Calcrl and Pdgfra. Data are expressed as means \pm SD from three independent experiments. All scale bars, $100 \mu \mathrm{m}$. FGF2, basic fibroblast growth factor; GFAP, glial fibrillary acidic protein; Ho, Hoechst 33342-stained nuclei; mNSCs, mouse neural stem cells; OLs, oligodendrocytes; OPCs, oligodendrocyte progenitor cells; PDGF-AA, platelet-derived growth factor-AA; SAG, smoothened agonist. ied in size and were positive for Sox1, Pax6, and Nestin within 5 days [17] (Fig. 1b).

Since oligodendrogenesis went through several differentiation stages characterized by distinct morphological and antigenic changes until final differentiation into myelin-forming OLs $[1,18]$, our next step was to pattern mNSCs into pre-OL lineage under well-defined condi-

Rapid and Efficient Differentiation of Rodent NSCs into OPCs tions. We transferred mNSCs onto a coated dish in the differentiation medium consisting of SHH, FGF2, and PDGF-AA to allow culture for 4 days. Neurospheres were laid down as adherent cells and gave rise to OPCs, which showed a typical bipolar or tripolar morphology and expressed transcription factors: Olig2, Nkx2.2, and Sox10 (Fig. 1c, d). The mNSC-derived OPCs represented a ho- 
mogenous cell population because they expressed OPC markers as high as $\sim 90 \%$ (Fig. 1e). In addition, the expandability of mNSC-derived OPCs was tested by a growth curve assay, indicating that mNSC-derived OPCs could expand into a homogenous population through at least eight passages, thus yielding a large cell number $\left(>10^{8}\right.$ cells, Fig. 1f). These results demonstrate that in the defined culture medium, OPCs can be rapidly produced from mNSCs.

In order to confirm the necessity of SHH signaling in the generation of OPCs from mNSCs [19], we replaced SHH with SAG, a chlorobenzothiophene-containing Hedgehog pathway agonist [20], in the medium. After treatment with $0.4 \mu \mathrm{M}$ SAG, FGF2, and PDGF-AA for 4 days, mNSCs underwent differentiation into OPCs, accompanied by downregulation of the expression of neuronal precursor genes, such as Sox1, Pax6, and Nestin, but upregulation of OL lineage promoters Olig2 and Nkx2.2 (Fig. $2 \mathrm{a}, \mathrm{b}$ ). As a rule, most of the oligodendroglial cells were born after neurons and astroglia in the CNS development [10]. The differentiated OPCs were doublestained with both Olig2 (a specific marker for OPCs) and the intermediate filament glial fibrillary acidic protein (GFAP), a specific marker for astrocytes [21]. We noted that the percentage of Olig2- and GFAP-positive cells in the total population was $91 \pm 3 \%$ and $1 \pm 1 \%$, respectively, which indicated that OPC marker expression (Olig2) was significantly higher than the latter value (Fig. 2c, d). This comparison suggested that addition of inducers SAG, FGF2, and PDGF-AA was very successful. RT-PCR analysis further indicated that mNSC-derived OPCs expressed the OL lineage-specific promoters and surface markers, such as Ascl1 (also known as Mash1), Olig1/2, Sox10, Calcrl, Pdgfra, and Cspg4 (also known as NG2), and that mNSC-derived OLs expressed high levels of transcription factors Olig1/2 and Sox10 (Fig. 2e). These results demonstrate that SAG, as a substitute for $\mathrm{SHH}$, could induce Hedgehog signaling activation in OPC generation and expansion.

\section{Differentiation of $m$ NSC-Derived OPCs into OLs}

within 3 Days

In the terminal differentiation of OPCs, T3 plays a key role in controlling OL maturation [22]. On the other hand, fibroblast growth factor and PDGF cooperate to promote rapid division of OPCs and to inhibit the further differentiation and maturation of OPCs [23]. To determine the differentiation ability of mNSC-derived OPCs into OLs, the OPCs were exposed to T3, SAG, Noggin, cAMP, IGF-1, and NT-3 [13], but not to FGF2 and PDGF-
AA (Fig. 3a). These conditions caused the cells to stop proliferation but to differentiate exclusively into OL-like cells with a round cell body and multiple processes over a time course of 2-4 days. Immature OLs did not express the myelinating surface marker MBP [3], but they were positive for MBP after 3-day induction (Fig. 3b, c). RTPCR data showed that the generated OLs strongly expressed specific myelin genes, such as myelin-associated oligodendrocytic basic protein, MBP, myelin protein Plp1/DM-20, myelin-associated glycoprotein, and myelin/OL glycoprotein (Fig. 3d), and also expressed high levels of Olig1/2 and Sox10 (Fig. 2e). The results suggest that mNSC-derived OPCs could differentiate into mature OLs within 3 days (total 7 days from the initial generation of mouse primary OPCs).

\section{Differentiation of $m N S C$-Derived OPCs into}

Astrocytes within 7 Days

OPCs, also called OL type 2 astrocyte progenitor cells, exhibit multipotential fates both in vivo and in vitro. They can differentiate into not only OLs but also astrocytes depending on culture conditions [24]. In this study, mNSCderived OPCs were also exposed to signaling cascades that specifically induce cells to differentiate into an alternative glial fate, namely astrocytes. Treatment of mNSCderived OPCs with $20 \mathrm{ng} / \mathrm{mL}$ BMP4 [25] over 5-7 days resulted in the majority of cells undergoing morphological changes and forming characteristic star-shaped glial cells with long fibrous unbranched processes. The formed cells expressed GFAP, indicative of differentiation into type 2 astrocyte-like cells (Fig. 3e).

As is known, type 2 astrocytes differ from type 1 astrocytes in morphology [26]. Additionally, astroglial lineage is the most abundant cell type in the CNS, which is generally technically easier for manipulation than neurons and OLs $[10,27]$. We attempted to induce differentiation toward astrocytes through culturing OPCs only with FBS but without growth factors. Interestingly, we observed that the majority of cells also differentiated toward GFAPpositive type 1 astrocyte-like cells with protoplasmic morphology (Fig. 3f), suggesting that mNSC-derived OPCs could give rise to both type 1 and type 2 astrocytelike cells in a certain medium within 7 days.

\section{Differentiation of rNSCs toward OL Lineage}

During embryo development, extracellular signals may have different effects on mice and rats [28]. To examine our designed protocol, we simultaneously induced rNSCs into OPCs and OLs with the same differentiation protocols. These rNSCs were isolated from E13.5 preg-
Li/Zheng/Chai/Lin/Zeng/Lu/Bian 

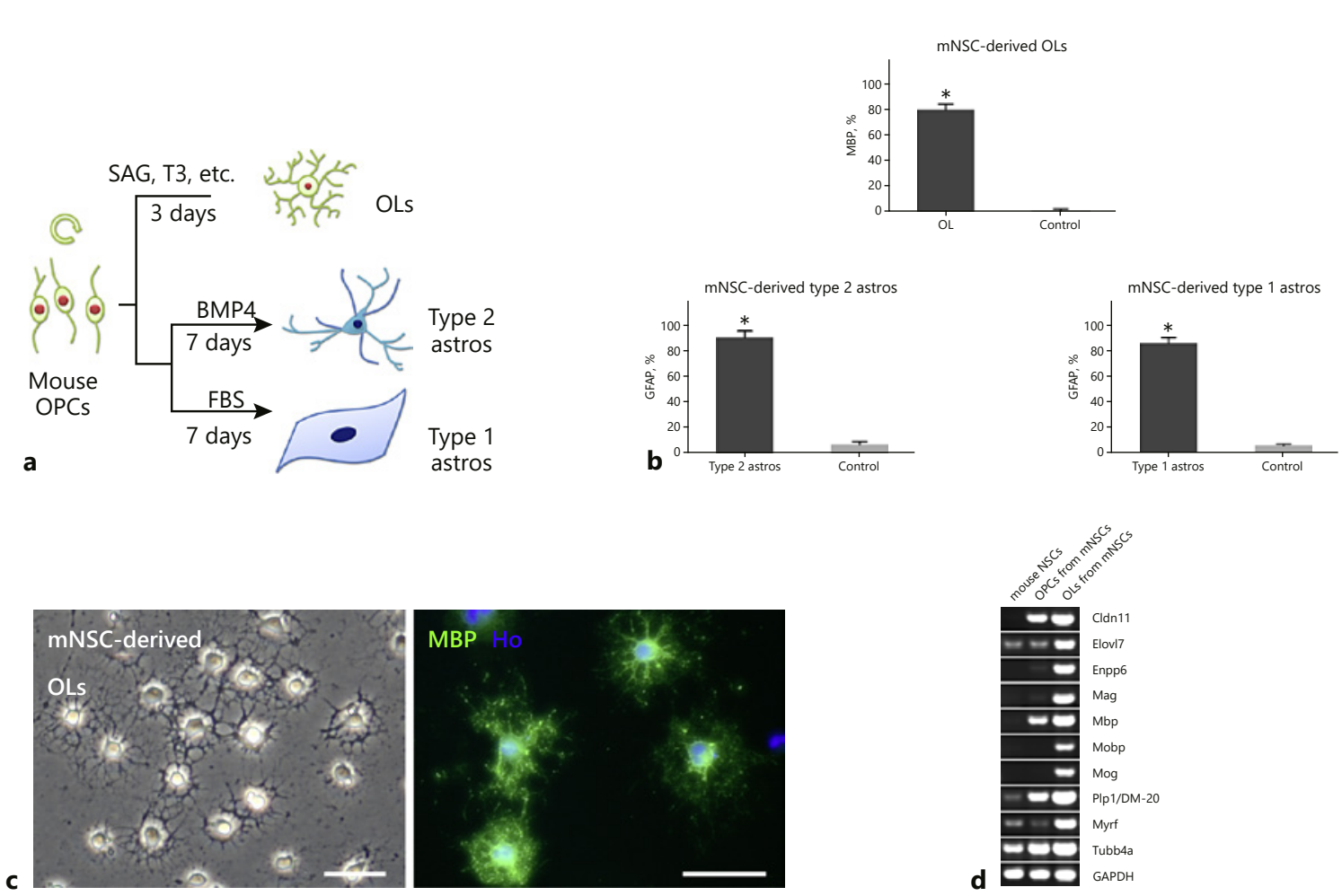

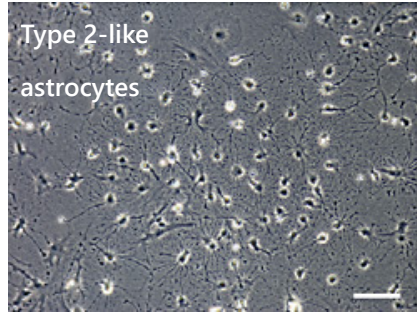

e

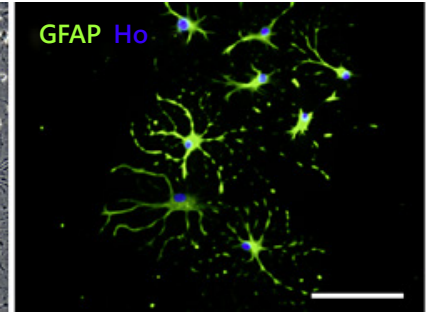

f
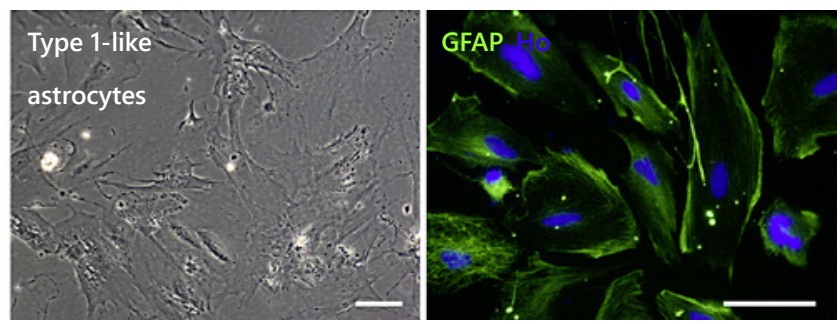

Fig. 3. Differentiation of mNSC-derived OPCs into mature OLs or astrocytes under defined conditions. a Schematic representation showing two alternative differentiation pathways of mNSC-derived OPCs into OLs or astrocytes (type 1 or 2). b Percentage of MBP- and GFAP-positive cells in alternative terminal protocol differentiation from mNSC-derived OPCs. Control medium was the OPC medium. ${ }^{*} p<0.05$. c Phase contrast and fluorescence images of mNSC-derived OPCs cultured in OL-inducing medium (T3, SAG, and other components excluding FGF2 and PDGF-AA) for 3 days, generating a mature OL population positive for mature OLs' marker MBP expressed in OLs' plasma membranes. d RTPCR analysis of mature OL marker genes in mNSCs, mNSC-derived OPCs, and OLs. mNSC-derived OLs had strong expression of specific myelin genes, such as myelin-associated oligodendro- cytic basic protein, MBP, Plp1/DM-20, myelin-associated glycoprotein, and myelin/OL glycoprotein. GAPDH was used as a loading control. e mNSC-derived OPCs differentiated into type 2 astrocytes under induction of BMP4. The majority of cells showed distinct morphological change and had GFAP expression. f mNSC-derived OPCs differentiated into type 1 astrocytes under induction of FBS. Scale bars, $50 \mu \mathrm{m}$ (c) and $100 \mu \mathrm{m}(\mathbf{e}, \mathbf{f})$. FBS, fetal bovine serum; FGF2, basic fibroblast growth factor; GFAP, glial fibrillary acidic protein; Ho, Hoechst 33342-stained nuclei; MBP, myelin basic protein; mNSCs, mouse neural stem cells; OLs, oligodendrocytes; OPCs, oligodendrocyte progenitor cells; PDGFAA, platelet-derived growth factor-AA; SAG, smoothened agonist; T3, triiodothyronine. 


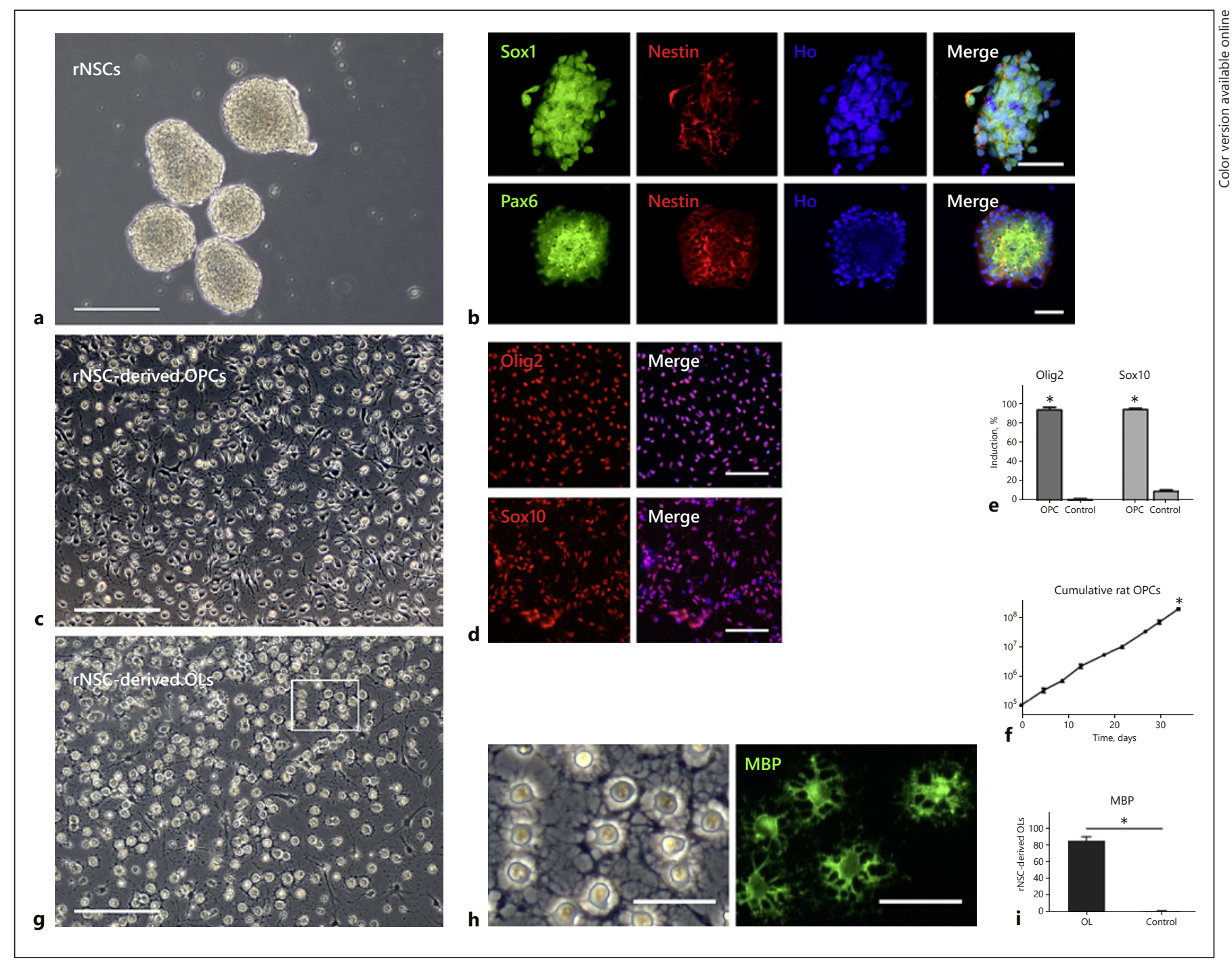

Fig. 4. Differentiation of rNSCs into OPCs and OLs. a Phase contrast image of rNSCs isolated from rat embryos. b Expanded rNSCs in vitro expressing NSC markers such as Sox1, Pax6, and Nestin. c Phase contrast image of OPCs derived from rNSCs under the defined medium consisting of SAG, FGF2, and PDGF-AA within 4 days. d Immunostaining of rNSC-derived OPCs with OPC markers Olig2 and Sox10. e Percentage of Olig2- and Sox10positive cells in the total population of rNSC-derived OPCs, which is significantly higher than the negative control (rNSCs treated with medium only with B27 and N2). f Cumulative growth curve of rNSC-derived OPCs over eight passages (P1-P8), P8 versus P1P7, respectively. $g$ Differentiation of rNSC-derived OPCs into mature OLs under the defined differentiation medium. $\mathbf{h}$ Immunos- taining of rNSC-derived OLs with the myelin marker MBP. The induction medium was the same as that for the generation of rNSC-derived OLs. i Percentage of MBP-positive cells in the differentiation of rNSC-derived OPCs with OL differentiation medium compared to the control, which was only treated with the OPC culture medium. Data are expressed as means \pm SD from three independent experiments. ${ }^{*} p<0.05$. Scale bars, $200 \mu \mathrm{m}$ (a, c, g), $100 \mu \mathrm{m}(\mathbf{d})$, and $50 \mu \mathrm{m}(\mathbf{b}, \mathbf{h})$. FGF2, basic fibroblast growth factor; Ho, Hoechst 33342-stained nuclei; MBP, myelin basic protein; NSC, neural stem cell; OLs, oligodendrocytes; OPCs, oligodendrocyte progenitor cells; PDGF-AA, platelet-derived growth factor-AA; rNSCs, rat neural stem cells; SAG, smoothened agonist. nant Sprague-Dawley rats. When the same procedure was carried out for rNSCs, the cells were induced to differentiate efficiently into OPCs within 4 days and then into OLs within an additional 3 days without specific modifi- cations to the protocol. The rNSCs isolated from rat embryos (Fig. 4a) could be expanded into neurospheres in vitro, and they expressed NSC markers such as Sox1, Pax6, and Nestin (Fig. 4b). rNSC-derived OPCs were gen-
86

Dev Neurosci 2019;41:79-93

DOI: $10.1159 / 000499364$
Li/Zheng/Chai/Lin/Zeng/Lu/Bian 


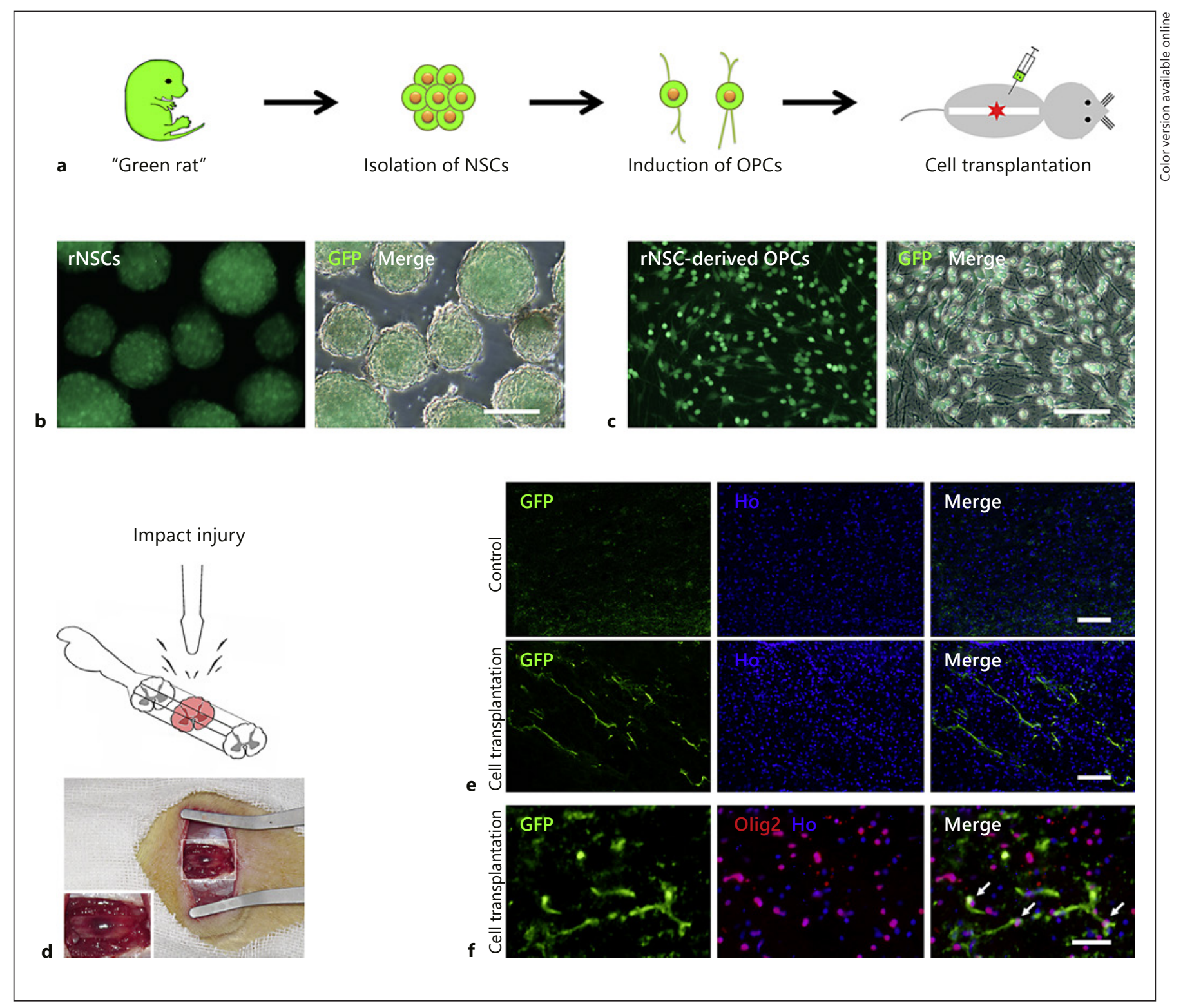

Fig. 5. Transplantation of rat OPCs into a rat model of spinal cord injury. a Schematic for OPC generation from NSCs isolated from rat GFP-positive embryos and transplantation of these OPCs into the injured spinal cord. b Bright-field and fluorescence images of rNSCs isolated from GFP-positive E13.5 embryo cortex. c Highly enriched GFP-positive OPCs induced from rNSCs under treatment with SAG, FGF2, and PDGF-AA. d Establishment of a rat model of spinal cord contusion in the 11th thoracic vertebra with the impactor. e After cell transplantation, rNSC-derived GFP
OPCs survived in the injured spinal cord. f Arrows indicate GFPpositive cells that also expressed the OL lineage marker Olig2 in the white matter. Scale bars, $100 \mu \mathrm{m}(\mathbf{b}, \mathbf{c}, \mathbf{e})$ and $50 \mu \mathrm{m}(\mathbf{f})$. E, embryonic day; FGF2, basic fibroblast growth factor; GFP, green fluorescent protein; Ho, Hoechst 33342-stained nuclei; NSCs, neural stem cells; OL, oligodendrocyte; OPCs, oligodendrocyte progenitor cells; PDGF-AA, platelet-derived growth factor-AA; rNSCs, rat neural stem cells; SAG, smoothened agonist.

ly higher than that for negative control (medium only with B27 and N2) (Fig. 4e). In addition, the expandability of mNSC-derived OPCs was tested by a cumulative growth curve, which indicated that rat OPCs could expand into a homogenous population through at least 


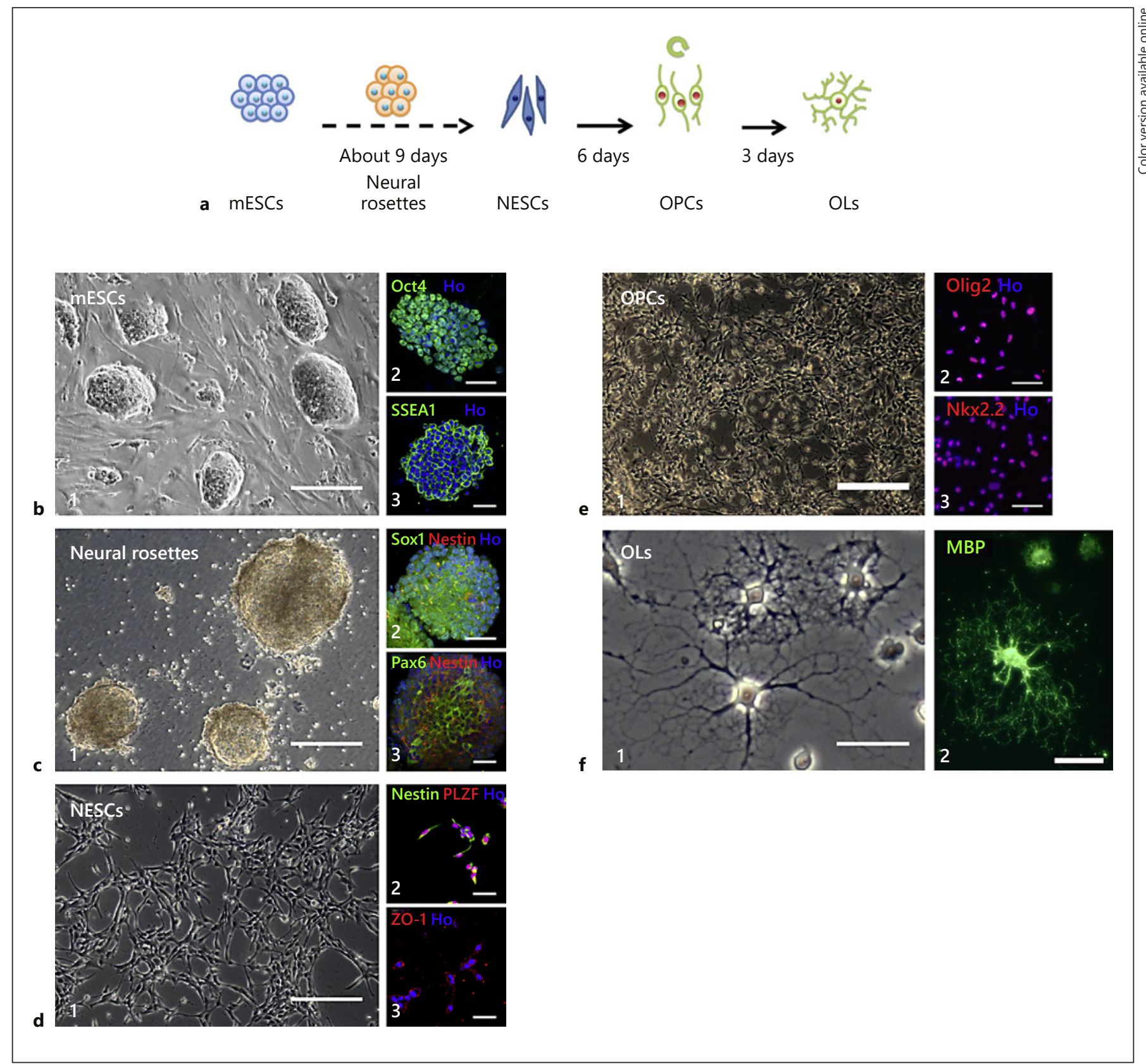

Fig. 6. Sequential differentiation of mESC-derived NESCs into OL lineage with a typical morphology and antigenic profile. a Experimental paradigm showing differentiation stages and time course of OLs from the mESC line. $\mathbf{b}$ At the beginning, mESCs were dissociated and cultured on a coated plate with neural differentiation medium. c On day 4, these single cells were formed into neural rosette-like colonies, which could be released and cultured into nonadherence neurospheres (see also online suppl. Fig. S1A) over a 4-day time course. $\mathbf{d}$ NESCs were dissociated from these neurospheres within a 1-day time course ( 9 days totally from the initial generation of the mouse primary ESCs). e By days 4-6, mESC-

derived NESCs were differentiated into OPCs with a bipolar or tripolar morphology and high expression of Olig2/Nkx2.2 under SAG, FGF2, and PDGF-AA treatments. f mESC-derived longterm self-renewing OPCs could be generated into terminal myelinating OLs. Scale bars, $100 \mu \mathrm{m}(\mathbf{b} 1, \mathbf{c 1}, \mathbf{d 1}, \mathbf{e 1})$ and $50 \mu \mathrm{m}(\mathbf{b 2}$, b3, c2, c3, d2, d3, e2, e3, f1, f2). ESCs, embryonic stem cells; FGF2, basic fibroblast growth factor; Ho, Hoechst 33342-stained nuclei; mESCs, mouse embryonic stem cells; NESCs, neuroepithelial stem cells; OLs, oligodendrocytes; OPCs, oligodendrocyte progenitor cells; PDGF-AA, platelet-derived growth factor-AA; SAG, smoothened agonist. 
Fig. 7. Schematic differentiation protocols of rapid generation of OPCs from various rodent NSCs. FBS, fetal bovine serum; FGF2, basic fibroblast growth factor; mESCs, mouse embryonic stem cells; mNSCs, mouse neural stem cells; NESCs, neuroepithelial stem cells; NSCs, neural stem cells; OLs, oligodendrocytes; OPCs, oligodendrocyte progenitor cells; PDGFAA, platelet-derived growth factor-AA; rNSCs, rat neural stem cells; SAG, smoothened agonist; T3, triiodothyronine.

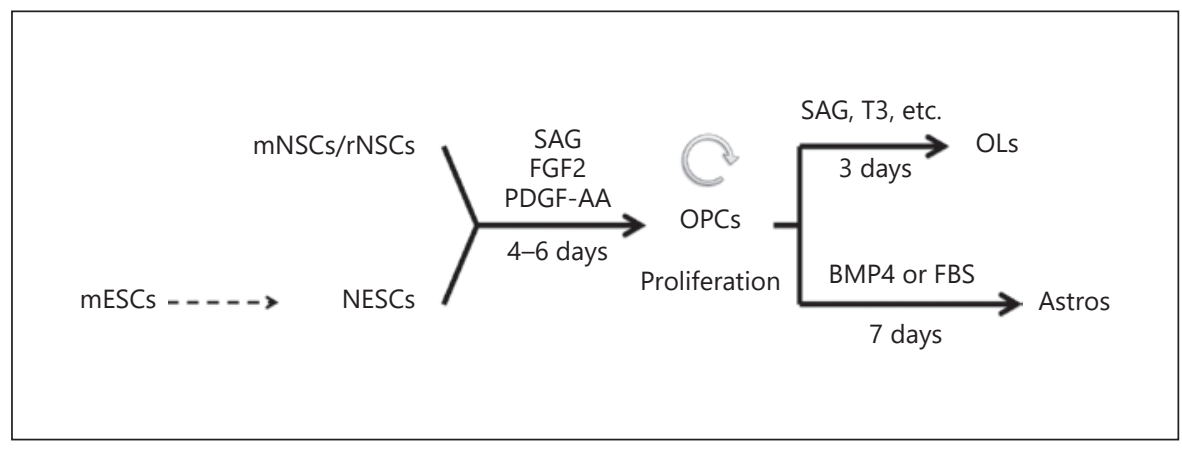

eight passages, yielding $10^{8}$ cells (Fig. 4f). Furthermore, rNSC-derived OPCs could differentiate into mature OLs under the defined differentiation medium (Fig. 4g), and they were positive for myelin marker MBP (Fig. 4h, i). These results demonstrate that OPCs and OLs could be rapidly and robustly generated from NSCs both in mice and rats.

Transplantation of GFP-Positive rNSC-Derived OPCs into the Injured Spinal Cord

The rat as a model of spinal cord injury was widely used for the study of nerve regeneration [29]. To verify our differentiation protocol for the application in cell transplantation in vivo, we established a rat spinal cord contusion model for OPC transplantation. The green OPCs induced from NSCs of embryonic transgenic Sprague-Dawley rats expressed GFP, enabling us to trace the survival of the transplanted cells (Fig. 5b, c). We used an impactor instrument to establish the rat spinal cord injury (Fig. 5d). Seven days after the injury in rats, we transplanted the expanded OPCs into a dorsal spinal cord contusion lesion of Sprague-Dawley rats. Five weeks after OPC transplantation, we found that GFP-positive transplanted cells survived in the injured spinal cord (Fig. 5e, f; arrows highlight some GFP-positive OPCs also coexpressing Olig2). These findings further indicate that transplanted OPCs, based on our protocol for oligodendroglial generation, have a therapeutic potential in the spinal cord injury model.

\section{Differentiation of mESC-Derived NESCs toward OL \\ Lineage}

NESCs, isolated from the rodent E10.5 caudal neural tube, have the ability to give rise to neural differentiation lineage: neurons, astrocytes, and OLs [30]. Previous work has also shown that NESCs could be derived from mESCs in vitro and exhibited a high similarity to NSCs in terms of extracellular signals and multipotent characters [31].
Treatment of mESCs with CHIR99021 and dual SMAD inhibitors [32,33] allowed the generation of NESCs from mESCs (including stages of neural rosettes and neurospheres) without forming embryoid bodies (online suppl. Fig. S1). Over a 9-day time course, NESCs exhibited a characteristic morphology and expressed markers including PLZF, Nestin, and ZO-1 (Fig. 6d). Then, we tried to turn the cell fate of NESCs to oligodendroglial lineage. Similar to the above-mentioned NSC-oligodendroglial differentiation, mESC-derived NESCs were exposed to SAG, FGF2, and PDGF-AA. After culture for 4-6 days, the cells showed bi- or tripolar morphology, coexpressed Olig2 and Nkx2.2 (both 90\% positive), and could be considered OPCs (Fig. 6e). The defined culture condition also maintained a large number of self-renewing OPCs (online suppl. Fig. S2). The obtained OPCs stopped expansion and differentiated into terminal OLs by using the same protocol (Fig. 6f). These results suggest that our protocol might be highly efficient in switching mNSCs, rNSCs, and mESC-derived NESCs to OL lineage (Fig. 7).

\section{Discussion}

OLs, the CNS myelinating cells, originate from OPCs in the embryonic brain and spinal cord. They could be generated in vitro from various NSCs [1]. For many years, the generation of OLs and OPCs was often used as a basis for in-depth studies of myelin-related disorders and cell replacement therapies [33-35]. Although many studies achieved some progress by using NSC- or pluripotent stem cell-derived OPCs $[7,8]$, the derived cells were mostly a heterogenous population of uncharacterized, tripotent neural precursors rather than pure OPCs. The purpose of this study was to test whether our differentiation protocol could allow a homogenous population of OPCs to be generated from rodent NSCs or mESC-derived NESCs. 
Environmental signaling via intercellular pathways may activate the expression of transcription factors in cell nuclei and may bind to specific DNA sequences to control cellular maturation. In the developing spinal cord, OPCs first emerge from the ventral ventricular zone of the neural tube in response to a secretory protein - SHH [23]. The graded SHH signaling from the notochord and floor plate establishes the expression in the defined domain by directly inducing transcriptional targets Olig1/2 and Nkx2.2 and repressing Pax6, Irx3, and Dbx1/2 [36-38], which is necessary and sufficient for the spatial and temporal specification of OPCs in the developing CNS. In addition, before their terminal differentiation into myelin formation, OLs go through the immature stage, and immature cells have proliferative capacities and migratory abilities involving fibroblast growth factor and PDGF pathways [39-41]. The fibroblast growth factors family regulates early development of OPCs. FGF2 appears to promote OPC proliferation from neural progenitor cells by upregulating Olig2 and PDGF receptor alpha (PDGFRa). Activation of PDGFRa kinase induces OPC proliferation by stimulating the MAP kinase, phosphatidylinositol 3-kinase, and phospholipase C gamma pathways $[42,43]$. Based on these findings, we induced neural precursors to differentiate into the OL lineage through activation of the $\mathrm{SHH}$ signaling pathway with either SHH or SAG and supplemented FGF2 and PDGF-AA as mitogens in the culture medium for OPC proliferation. The culture conditions consisting of SAG, FGF2, and PDGF-AA rapidly drove mNSCs, rNSCs, and mESC-derived NESCs into OPCs, as evidenced by high expression of Olig 2 and $\mathrm{Nkx} 2.2$ [44], but no expression of GFAP by the cells, thus causing a shift of the fate of these multipotent cells toward an oligodendroglial fate. Our screening experiment, by adding some key factors responsible for OPC development in vivo, confirmed that combining three factors of PDGF-AA, FGF2, and SHH or SHH agonist (SAG) could efficiently differentiate rodent NSCs into pure committed OPCs, while other combinations such as NT3, PDGF-AA, and FGF2 as reported [45] could not reach such a high population of committed OPCs (data not shown).

In the transition of immature OPCs to their terminal destination of myelination, fetal and postnatal serum thyroid hormone levels (such as T3) played an important role both in vivo and in vitro $[46,47]$. Addition of some growth factors (such as PDGF-AA and FGF2) could enhance expansion and maintain cell proliferation, but could not directly promote OPC maturation by repressing the function or expression of differentiation-promoting factors such as Sox10, Myrf, and Sip1 [48]. Traditional maturation of mouse OPCs by adding only T3 could count for $50 \%$ conversion of OPCs into OLs [49]. According to most of the recently published methods of OPC maturation, it was a tedious process, which took 10 days or more to mature in vitro $[6,35]$. Therefore, our method showed that by withdrawing FGF2 and PDGF-AA and adding T3 together with SAG, Noggin, cAMP, IGF-1, and NT-3, OPCs could further differentiate into MBP-positive OLs within 3 days. Our method indicated that besides the key factors such as T3 and SAG, each additive such as Noggin, cAMP, IGF-1, and NT3 was indispensable to accelerate OPC maturation. Such combined components in differentiation could generate OLs in high yield and high purity as well. Noggin, a bone morphogenetic protein antagonist, was reported to act downstream of Wnt and SHH to antagonize BMP4 in somite patterning during embryo development $[50,51]$. It played an important role in the regulation of Olig2 so as to direct OPCs toward OLs and prevent OPC differentiation toward astrocytes [12]. cAMP has its important role in axon formation via a cAMP pathway in promoting myelination [52]. IGF-1 was reported in promoting NSC differentiation into OLs via the ERK1/2 pathway when differentiation of IGF-1 transfected spinal cord-derived NSCs into OLs [53]. Furthermore, NT3 was reported to promote differentiation of OPCs into OLs when scientists investigated purine-crosslinked injectable chitosan sponges' promotion in OPC attachment and differentiation [34]. Worth mentioning is that OPCs obtained under our current method could be further differentiated into both type 1 and type 2 astrocytes in high efficiency and high purity by adding FBS or BMP4, respectively [54]. Despite recent optimizations [55-57], these protocols remain inefficient and variable in terms of OL or astrocyte yield. Most importantly, these protocols require either very long differentiation process to generate mature glial cells such as OLs or astrocytes [58], or accelerate the differentiation process by overexpression of key transcription factors such as Sox 10 or NFIA with virus $[56,57]$. Comparing it to the most recent protocols [55, $56]$, our method is a naturally occurring process with high differentiation efficiency and no need to alter the genome by virus $[55,57]$, which provided a reliable safe way leading toward drug screening and cellular-based therapy.

In the nucleus, dynamic and restricted control of the transcriptional network, including Ascl1/Mash1, 
Olig1/2, Sox10, and Myrf, undergoes several differentiation stages for oligodendrogenesis. Ascl1/Mash1 is an early neural transcription factor which induces expression of PDGFRa by operating in genetic interaction with Olig2 [59], the most essential transcriptional regulator in the whole oligodendroglial differentiation. Collaboration of Olig2 and Nkx2.2 (a key timing regulator for OL differentiation) switches the cell fate to early oligodendroglial lineage [60]. Olig2 also regulates Sox10 [61], which is a direct activator to promote Myrf expression [62]. In the final phase of oligodendroglial maturation, Myrf coordinates with Olig1 and Sox10 and mediates myelin gene expression followed by terminal formation $[3,63]$. From transcriptional levels, the induced cells upregulated transcription factor Ascl1/Mash1, Olig1/2, and Sox 10 after treatment with SAG, FGF2, and PDGFAA. At further myelin differentiation, these MBP-positive OLs could downregulate Ascl1/Mash1 by terminal maturation, maintained Olig $1 / 2$, and gradually upregulated Sox10, Myrf, and other myelin genes.

In summary, we postulate that precise control of these signals would allow for the routine and specific generation of sufficient scalable OPCs from NSCs, which might provide therapeutic application in vivo. All those findings in our study presented not only a precise signaling pathway to control differentiation, but also extended its potential therapeutic application for the treatment of spinal cord injury.

\section{Acknowledgments}

This work was supported by the National Key Research \& Development Program and National Natural Science Foundations of China (2017YFC1200204 and 31670171), the Neural Regeneration Co-Innovation Center of Jiangsu Province, the Basic Research of Jiangsu Education Department (12KJA3100031), the Priority Academic Program Development (PAPD) of Jiangsu Higher Education Institutions and Nantong Science and Technology grant (MS12015055), and the student innovation project of Central South University (2018zzts231).

\section{Statement of Ethics}

All experimental procedures involving animals were conducted as per the institutional animal care guidelines of Nantong University and were approved ethically by the Jiangsu administration committee for experimental animals, China.

\section{Disclosure Statement}

The authors declare that there is no conflict of interest regarding the publication of this paper.

\section{Author Contributions}

J. Bian conceived and designed the experiments. S. Li, J. Zheng, and L. Chai performed the experiments. S. Li analyzed the data. M. Lin and J. Lu contributed the materials, models, and analysis tools. S. Li and R. Zeng wrote the paper. J. Lu and J. Bian provided financial support.

\section{References}

1 Miller RH. Regulation of oligodendrocyte development in the vertebrate CNS. Prog Neurobiol. 2002 Aug;67(6):451-67.

2 Lee JB, Affeldt BM, Gamboa Y, Hamer M, Dunn JF, Pardo AC, et al. Repeated pediatric concussions evoke long-term oligodendrocyte and white matter microstructural dysregulation distant from the injury. Dev Neurosci. 2018;40(4):358-75.

3 Emery B. Regulation of oligodendrocyte differentiation and myelination. Science. 2010 Nov;330(6005):779-82.

4 Li JY, Xie W, Strong JA, Guo QL, Zhang JM. Mechanical hypersensitivity, sympathetic sprouting, and glial activation are attenuated by local injection of corticosteroid near the lumbar ganglion in a rat model of neuropathic pain. Reg Anesth Pain Med. 2011 Jan-Feb; 36(1):56-62.

5 Chandran S, Compston A. Neural stem cells as a potential source of oligodendrocytes for myelin repair. J Neurol Sci. 2005 Jun;233(12):179-81.

Rapid and Efficient Differentiation of Rodent NSCs into OPCs
6 Neman J, de Vellis J. A method for deriving homogenous population of oligodendrocytes from mouse embryonic stem cells. Dev Neurobiol. 2012 Jun;72(6):777-88.

7 Wang Z, Sheng C, Li T, Teng F, Sang L, Cao $\mathrm{F}$, et al. Generation of tripotent neural progenitor cells from rat embryonic stem cells. J Genet Genomics. 2012 Dec;39(12):643-51.

8 Medina-Rodríguez EM, Arenzana FJ, Bribián A, de Castro F. Protocol to isolate a large amount of functional oligodendrocyte precursor cells from the cerebral cortex of adult mice and humans. PLoS One. 2013 Nov; 8(11):e81620.

9 Wang S, Bates J, Li X, Schanz S, ChandlerMilitello D, Levine C, et al. Human iPSC-derived oligodendrocyte progenitor cells can myelinate and rescue a mouse model of congenital hypomyelination. Cell Stem Cell. 2013 Feb;12(2):252-64.

10 Liu H, Zhang SC. Specification of neuronal and glial subtypes from human pluripotent stem cells. Cell Mol Life Sci. 2011 Dec;68(24): 3995-4008.
11 Richardson WD, Pringle NP, Yu WP, Hall AC. Origins of spinal cord oligodendrocytes: possible developmental and evolutionary relationships with motor neurons. Dev Neurosci. 1997;19(1):58-68.

12 Richardson WD, Kessaris N, Pringle N. Oligodendrocyte wars. Nat Rev Neurosci. 2006 Jan; 7(1):11-8.

13 Najm FJ, Zaremba A, Caprariello AV, Nayak S, Freundt EC, Scacheri PC, et al. Rapid and robust generation of functional oligodendrocyte progenitor cells from epiblast stem cells. Nat Methods. 2011 Sep;8(11):957-62.

14 Tropepe V, Sibilia M, Ciruna BG, Rossant J, Wagner EF, van der Kooy D. Distinct neural stem cells proliferate in response to EGF and FGF in the developing mouse telencephalon. Dev Biol. 1999 Apr;208(1):166-88.

15 Reynolds BA, Tetzlaff W, Weiss S. A multipotent EGF-responsive striatal embryonic progenitor cell produces neurons and astrocytes. J Neurosci. 1992 Nov;12(11):4565-74. 
16 Cera AA, Cacci E, Toselli C, Cardarelli S, Bernardi A, Gioia R, et al. Egr-1 maintains NSC proliferation and its overexpression counteracts cell cycle exit triggered by the withdrawal of epidermal growth factor. Dev Neurosci. 2018;40(3):223-33.

17 Chen G, Ma J, Shatos MA, Chen H, Cyr D, Lashkari K. Application of human persistent fetal vasculature neural progenitors for transplantation in the inner retina. Cell Transplant. 2012;21(12):2621-34.

18 Naruse M, Shibasaki K, Shimauchi-Ohtaki H, Ishizaki Y. Microglial Activation Induces Generation of Oligodendrocyte Progenitor Cells from the Subventricular Zone after Focal Demyelination in the Corpus Callosum. Dev Neurosci. 2018;40(1):54-63.

19 Jiang W, Ishino Y, Hashimoto H, Keino-Masu K, Masu M, Uchimura K, et al. Sulfatase 2 Modulates Fate Change from Motor Neurons to Oligodendrocyte Precursor Cells through Coordinated Regulation of Shh Signaling with Sulfatase 1. Dev Neurosci. 2017;39(5): 361-74.

20 Asaoka Y, Kanai F, Ichimura T, Tateishi K, Tanaka Y, Ohta M, et al. Identification of a suppressive mechanism for Hedgehog signaling through a novel interaction of Gli with 143-3. J Biol Chem. 2010 Feb;285(6):4185-94.

21 Liu F, Liao F, Li W, Han Y, Liao D. Progesterone alters Nogo-A, GFAP and GAP-43 expression in a rat model of traumatic brain injury. Mol Med Rep. 2014 Apr;9(4):1225-31.

22 Baxi EG, Schott JT, Fairchild AN, Kirby LA, Karani R, Uapinyoying P, et al. A selective thyroid hormone $\beta$ receptor agonist enhances human and rodent oligodendrocyte differentiation. Glia. 2014 Sep;62(9):1513-29.

$23 \mathrm{He} \mathrm{L,} \mathrm{Lu} \mathrm{QR.} \mathrm{Coordinated} \mathrm{control} \mathrm{of} \mathrm{oligo-}$ dendrocyte development by extrinsic and intrinsic signaling cues. Neurosci Bull. 2013 Apr;29(2):129-43.

24 Kondo T, Raff M. Oligodendrocyte precursor cells reprogrammed to become multipotential CNS stem cells. Science. 2000 Sep; 289(5485):1754-7.

25 Samanta J, Kessler JA. Interactions between ID and OLIG proteins mediate the inhibitory effects of BMP4 on oligodendroglial differentiation. Development. 2004 Sep;131(17): 4131-42.

26 Raff MC, Abney ER, Cohen J, Lindsay R, Noble M. Two types of astrocytes in cultures of developing rat white matter: differences in morphology, surface gangliosides, and growth characteristics. J Neurosci. 1983 Jun; 3(6):1289-300.

27 Kessaris N, Pringle N, Richardson WD. Specification of CNS glia from neural stem cells in the embryonic neuroepithelium. Philos Trans R Soc Lond B Biol Sci. 2008 Jan;363(1489): 71-85.

28 Li P, Tong C, Mehrian-Shai R, Jia L, Wu N, Yan Y, et al. Germline competent embryonic stem cells derived from rat blastocysts. Cell. 2008 Dec;135(7):1299-310.
29 Yartsev MM. The emperor's new wardrobe: rebalancing diversity of animal models in neuroscience research. Science. 2017 Oct; 358(6362):466-9.

30 Kalyani A, Hobson K, Rao MS. Neuroepithelial stem cells from the embryonic spinal cord: isolation, characterization, and clonal analysis. Dev Biol. 1997 Jun;186(2):202-23.

31 Merkle FT, Alvarez-Buylla A. Neural stem cells in mammalian development. Curr Opin Cell Biol. 2006 Dec;18(6):704-9.

32 Bian J, Zheng J, Li S, Luo L, Ding F. Sequential differentiation of embryonic stem cells into neural Epithelial-Like stem cells and oligodendrocyte progenitor cells. PLoS One. 2016 May;11(5):e0155227.

33 Chambers SM, Fasano CA, Papapetrou EP, Tomishima M, Sadelain M, Studer L. Highly efficient neural conversion of human ES and iPS cells by dual inhibition of SMAD signaling. Nat Biotechnol. 2009 Mar;27(3):275-80.

34 Mekhail M, Almazan G, Tabrizian M. Purine-crosslinked injectable chitosan sponges promote oligodendrocyte progenitor cells' attachment and differentiation. Biomater Sci. 2015 Feb;3(2):279-87.

35 Sun Y, Xu CC, Li J, Guan XY, Gao L, Ma LX, et al. Transplantation of oligodendrocyte precursor cells improves locomotion deficits in rats with spinal cord irradiation injury. PLoS One. 2013;8(2):e57534.

36 Nery S, Wichterle H, Fishell G. Sonic hedgehog contributes to oligodendrocyte specification in the mammalian forebrain. Development. 2001 Feb;128(4):527-40.

37 Martí E, Bovolenta P. Sonic hedgehog in CNS development: one signal, multiple outputs. Trends Neurosci. 2002 Feb;25(2):8996.

38 Nishi Y, Zhang X, Jeong J, Peterson KA, Vedenko A, Bulyk ML, et al. A direct fate exclusion mechanism by Sonic hedgehog-regulated transcriptional repressors. Development. 2015 Oct;142(19):3286-93.

39 Tsai HH, Niu J, Munji R, Davalos D, Chang J, Zhang $\mathrm{H}$, et al. Oligodendrocyte precursors migrate along vasculature in the developing nervous system. Science. 2016 Jan; 351(6271):379-84

40 Vora P, Pillai PP, Zhu W, Mustapha J, Namaka MP, Frost EE. Differential effects of growth factors on oligodendrocyte progenitor migration. Eur J Cell Biol. 2011 Aug; 90(8):649-56.

41 Wan X, Zhang L, Jiang B. Role of endothelin $\mathrm{B}$ receptor in oligodendroglioma proliferation and survival: in vitro and in vivo evidence. Mol Med Rep. 2014 Jan;9(1):229-34.

42 Naruse M, Nakahira E, Miyata T, Hitoshi S, Ikenaka K, Bansal R. Induction of oligodendrocyte progenitors in dorsal forebrain by intraventricular microinjection of FGF-2. Dev Biol. 2006 Sep;297(1):262-73.
43 McKinnon RD, Waldron S, Kiel ME. PDGF alpha-receptor signal strength controls an RTK rheostat that integrates phosphoinositol $3^{\prime}$-kinase and phospholipase Cgamma pathways during oligodendrocyte maturation. J Neurosci. 2005 Apr;25(14):3499-508.

44 Fancy SP, Zhao C, Franklin RJ. Increased expression of Nkx2.2 and Olig2 identifies reactive oligodendrocyte progenitor cells responding to demyelination in the adult CNS. Mol Cell Neurosci. 2004 Nov;27(3):247-54.

45 Neri M, Maderna C, Ferrari D, Cavazzin C, Vescovi AL, Gritti A. Robust generation of oligodendrocyte progenitors from human neural stem cells and engraftment in experimental demyelination models in mice. PLoS One. 2010 Apr;5(4):e10145.

46 Lee JY, Petratos S. Thyroid hormone signaling in oligodendrocytes: from extracellular transport to intracellular signal. Mol Neurobiol. 2016 Nov;53(9):6568-83.

47 Younes-Rapozo V, Berendonk J, Savignon T, Manhães AC, Barradas PC. Thyroid hormone deficiency changes the distribution of oligodendrocyte/myelin markers during oligodendroglial differentiation in vitro. Int $J$ Dev Neurosci. 2006 Nov;24(7):445-53.

48 Huang H, Zhao XF, Zheng K, Qiu M. Regulation of the timing of oligodendrocyte differentiation: mechanisms and perspectives. Neurosci Bull. 2013 Apr;29(2):155-64.

49 Wang Y, Tan ZB, Qin LM, Yu YQ. Simplified protocol for isolation of multipotential NG2 cells from postnatal mouse. J Neurosci Methods. 2013 Oct;219(2):252-61.

50 Hirsinger E, Duprez D, Jouve C, Malapert P, Cooke J, Pourquié O. Noggin acts downstream of Wnt and Sonic Hedgehog to antagonize BMP4 in avian somite patterning. Development. 1997 Nov;124(22):4605-14.

51 Dettman RW, Birch D, Fernando A, Kessler JA, Dizon ML. Targeted knockdown of bone morphogenetic protein signaling within neural progenitors protects the brain and improves motor function following postnatal Hypoxia-Ischemia. Dev Neurosci. 2018; 40(1):23-38.

52 Malone M, Gary D, Yang IH, Miglioretti A, Houdayer T, Thakor N, et al. Neuronal activity promotes myelination via a cAMP pathway. Glia. 2013 Jun;61(6):843-54.

53 Shi B, Ding J, Liu Y, Zhuang X, Zhuang X, Chen $X$, et al. ERK1/2 pathway-mediated differentiation of IGF-1-transfected spinal cordderived neural stem cells into oligodendrocytes. PLoS One. 2014 Aug;9(8):e106038.

54 Peng T, Zhu G, Dong Y, Zeng J, Li W, Guo W, et al. BMP4: A possible key factor in differentiation of auditory Neuron-Like cells from Bone-Derived mesenchymal stromal cells. Clin Lab. 2015;61(9):1171-8.

55 García-León JA, Kumar M, Boon R, Chau D, One J, Wolfs E, et al. SOX10 single transcription factor-based fast and efficient generation of oligodendrocytes from human pluripotent stem cells. Stem Cell Reports. 2018 Feb;10(2): 655-72. 
56 Douvaras P, Wang J, Zimmer M, Hanchuk S, O'Bara MA, Sadiq S, et al. Efficient generation of myelinating oligodendrocytes from primary progressive multiple sclerosis patients by induced pluripotent stem cells. Stem Cell Reports. 2014 Aug;3(2):250-9.

57 Li X, Tao Y, Bradley R, Du Z, Tao Y, Kong L, et al. Fast generation of functional subtype astrocytes from human pluripotent stem cells. Stem Cell Reports. 2018 Oct;11(4):998-1008.

58 Madhavan M, Nevin ZS, Shick HE, Garrison E, Clarkson-Paredes C, Karl M, et al. Induction of myelinating oligodendrocytes in human cortical spheroids. Nat Methods. 2018 Sep;15(9):700-6.
59 Parras CM, Hunt C, Sugimori M, Nakafuku M, Rowitch D, Guillemot F. The proneural gene Mash1 specifies an early population of telencephalic oligodendrocytes. J Neurosci. 2007 Apr;27(16):4233-42.

60 Zhou Q, Choi G, Anderson DJ. The bHLH transcription factor Olig2 promotes oligodendrocyte differentiation in collaboration with Nkx2.2. Neuron. 2001 Sep;31(5):791807.

61 Küspert M, Hammer A, Bösl MR, Wegner M. Olig2 regulates Sox 10 expression in oligodendrocyte precursors through an evolutionary conserved distal enhancer. Nucleic Acids Res. 2011 Mar;39(4):1280-93.
62 Hornig J, Fröb F, Vogl MR, Hermans-Borgmeyer I, Tamm ER, Wegner M. The transcription factors Sox 10 and Myrf define an essential regulatory network module in differentiating oligodendrocytes. PLoS Genet. 2013 Oct;9(10):e1003907.

63 Emery B, Lu QR. Transcriptional and epigenetic regulation of oligodendrocyte development and myelination in the central nervous system. Cold Spring Harb Perspect Biol. 2015 Jul;7(9):a020461. 